\title{
Centralization of Health Care to Facilitate Greater Use of Nephron-Sparing Surgery for Localized Renal Tumors: Identifying Appropriate Health Care Delivery
}

\author{
Jeffrey C. Morrison, $\mathrm{MD}^{1}$, Boris Gershman, $\mathrm{MD}^{2}$, Badrinath Konety, MD, $\mathrm{MBA}^{3}$, Nicholas Cost, $\mathrm{MD}^{1,4}$, and \\ Simon P. Kim, MD, MPH ${ }^{1,5}$ \\ ${ }^{1}$ Division of Urology, Medical Center, University of Colorado, Aurora, CO; ${ }^{2}$ Division of Urology, Beth Israel Deaconess \\ Medical Center, Harvard Medical School, Boston, MA; ${ }^{3}$ Department of Urology, University of Minnesota, Minneapolis, \\ MN; ${ }^{4}$ Division of Pediatric Urology, Children's Hospital Colorado, Aurora, CO; ${ }^{5}$ Cancer Outcomes Public Policy and \\ Effectiveness Research (COPPER) Center, Yale University, New Haven, CT
}

Clinical practice guidelines have endorsed the principles of nephron-sparing surgery (NSS) to achieve comparable oncologic outcomes compared with radical nephrectomy $(\mathrm{RN})$, while minimizing the morbidity from chronic kidney disease. ${ }^{1-3}$ Indeed, the American Urological Association (AUA) recently further reinforced 'prioritizing' partial nephrectomy $(\mathrm{PN})$, in particular for clinical T1a $(<4 \mathrm{~cm})$ renal tumors, and limiting the RN for a specific set of criteria (higher complexity renal tumors with absence of pre-existing chronic kidney disease and proteinuria). ${ }^{2}$ In response to the greater emphasis in NSS to clinical practice guidelines attributable to the growing evidence regarding similar oncologic control, population-based studies have demonstrated greater use of PN over time. ${ }^{4-6}$

Efforts by key stakeholders, surgeons, and patients have now focused on identifying key characteristics of health care delivery to achieve higher quality, guideline-concordant health care and better patient health outcomes. In this issue of Annals of Surgical Oncology, Flegar et al. elucidated the trends in PN and RN for localized renal tumors in Germany and the US, and identified key hospital-related factors associated with NSS using administrative data from national administrative data and cancer registries from each

\footnotetext{
(C) Society of Surgical Oncology 2020
}

First Received: 30 November 2019;

Published Online: 23 January 2020

S. P. Kim, MD, MPH

e-mail: simkim@me.com; simon.kim@cuanshutz.edu country. ${ }^{7}$ Greater use of NSS for localized renal cell carcinoma (RCC) for both countries represented a key finding from this study, where in fact PN now constitutes a majority of renal surgery for localized $\mathrm{T} 1$ renal tumors from the cancer registries. Furthermore, the study also established that hospitals with higher annual surgical volume and access to robotic surgical systems were both clearly associated with NSS for RCC. Against this backdrop, the surgical approach influenced the type of renal surgery performed in Germany and the US, where open PN and RN rapidly declined counterbalanced against an expansion of minimally invasive surgery (MIS). More specifically, robotic surgery to achieve NSS became the predominant approach for localized renal tumors in the US. Although modest adoption of both MIS approaches for localized renal tumors was observed in Germany, approximately $70 \%$ and $80 \%$ of German patients received open $\mathrm{PN}$ and RN for localized RCC, respectively.

The central findings from this study have health care policy implications in achieving the goals of identifying and delivering high-quality health care where the appropriately selected patient diagnosed with a $\mathrm{T} 1$ renal tumor undergoes NSS. First, it has been well-documented that higher hospital and surgeon volume are clearly associated with greater utilization of appropriate complex cancer surgery, including PN, with better outcomes with less complications and lower health care costs and operative mortality. ${ }^{5,8,9}$ As a result, efforts to regionalize health care to higher-volume hospitals for patients with localized renal masses have been one consequence of achieving more NSS on a population-level. It is essential to acknowledge that 
these studies included by Flegar et al. rely on claims data that do not provide any information regarding tumor complexity or location. Indeed, a plausible criticism of claims data from cancer registries or insurance databases is the lack of any of these key clinical data regarding tumor complexity and location that greatly inform the safe and appropriate use of PN. While higher-volume hospitals and surgeons may in fact have higher utilization of NSS, it is also possible that patients undergoing $\mathrm{RN}$ at lower-volume hospitals have more centrally located or higher complexity tumors in the context of a normal contralateral kidney, and thus do indeed receive the clinically appropriate treatment.

Second, the national variation in the ability to successfully implement widespread robotic surgical systems across Germany and the US provide a salient contrast on how new surgical advanced technology, in particular robotic surgery, impact the use of NSS for localized RCC. ${ }^{7}$ While both countries continued to have a gradual increase in PN, with a decrease in RN over time, the largest increases in NSS in the US were attributable to robotic surgery. Other studies have supported the association of higher utilization of PN and robotic surgery where this advanced surgical technology now represents the majority of surgical approaches for localized T1 renal tumors. ${ }^{10}$ However, to date, there are limited level 1 data assessing the comparative effectiveness of robotic, laparoscopic, and open PN. Furthermore, robotic surgery, compared with open and laparoscopic surgery, has been associated with higher health care costs in the US. ${ }^{11}$ Another potential concern regarding the widespread adoption of robotic surgery for localized $\mathrm{RN}$ is the overtreatment of small renal masses that may be appropriately managed by active surveillance. ${ }^{12}$

In summary, this retrospective comparison study describes the increasing prevalence of NSS in Germany and the US, and also highlights several hospital-related factors positively associated with NSS and higher surgical volume and access to a robot. The findings thus suggest that patients may receive differing surgical care for renal tumors based on hospital characteristics or resources. In an effort to optimize guideline-concordant, high-value care, these data support further regionalization of renal surgical care to hospitals with higher surgical volume and, to some degree, those with the presence of robotic surgical systems. Yet, it is essential to acknowledge the inherent weaknesses in the granularity of claims data due to tumor complexity or location. Indeed, prior research has yet to define what the optimal rate of PN should be since medical decision making is somewhat subjective and limited data exist on the national rates of complexity for renal tumors. Furthermore, centralization of care to high-volume hospitals with robotic surgical systems may have unintended consequences of surgically treating renal tumors that could be managed by active surveillance. Therefore, further dissemination and implementation research is needed to address how we optimize medical decision making to discern which patients would be optimally treated with PN, $\mathrm{RN}$, and active surveillance. ${ }^{13}$

DISCLOSURES Dr. Simon P. Kim is supported by an R01 grant from the National Cancer Institute (MD12-003), and the Schramm Foundation. The other authors have nothing to disclose.

\section{REFERENCES}

1. Albiges L, Powles T, Staehler M, et al. Updated European Association of Urology guidelines on renal cell carcinoma: immune checkpoint inhibition is the new backbone in first-line treatment of metastatic clear-cell renal cell carcinoma. Eur Urol. 2019;76(2):151-156.

2. Campbell S, Uzzo RG, Allaf ME, et al. Renal mass and localized renal cancer: AUA guideline. J Urol. 2017;198(3):520-529.

3. Motzer RJ, Jonasch E, Agarwal N, et al. Kidney cancer, version 2.2017, NCCN clinical practice guidelines in oncology. $J$ Natl Compr Canc Netw. 2017;15(6):804-834.

4. Alameddine M, Koru-Sengul T, Moore KJ, et al. Trends in utilization of robotic and open partial nephrectomy for management of cT1 renal masses. Eur Urol Focus. 2019;5(3):482-487.

5. Kim SP, Shah ND, Weight CJ, et al. Contemporary trends in nephrectomy for renal cell carcinoma in the United States: results from a population based cohort. J Urol. 2011;186(5):1779-1785.

6. Tan HJ, Norton EC, Ye Z, Hafez KS, Gore JL, Miller DC. Longterm survival following partial vs radical nephrectomy among older patients with early-stage kidney cancer. JAMA. 2012;307(15):1629-1635.

7. Flegar L, Groeben C, Koch R, et al. Trends in renal tumor surgery in the United States and Germany between 2006 and 2014: organ preservation rate is improving. Ann Surg Oncol. 2019. https://doi. org/10.1245/s10434-019-08108-x.

8. Birkmeyer JD, Stukel TA, Siewers AE, Goodney PP, Wennberg DE, Lucas FL. Surgeon volume and operative mortality in the United States. N Engl J Med. 2003;349(22):2117-2127.

9. Tan HJ, Wolf JS Jr, Ye Z, Hafez KS, Miller DC. Population level assessment of hospital based outcomes following laparoscopic versus open partial nephrectomy during the adoption of minimally invasive surgery. J Urol. 2014;191(5):1231-1237.

10. Simone G, De Nunzio C, Ferriero M, et al. Trends in the use of partial nephrectomy for cT1 renal tumors: analysis of a 10-yr European multicenter dataset. Eur $J$ Surg Oncol. 2016;42(11):1729-1735.

11. Gershman B, Bukavina L, Chen Z, et al. The association of robotassisted versus pure laparoscopic radical nephrectomy with perioperative outcomes and hospital costs. Eur Urol Focus. 2018. https://doi.org/10.1016/j.euf.2018.10.004.

12. Shah PH, Alom MA, Leibovich BC, et al. The temporal association of robotic surgical diffusion with overtreatment of the small renal mass. J Urol. 2018;200(5):981-988.

13. Kim SP, Campbell SC, Gill I, et al. Collaborative review of risk benefit trade-offs between partial and radical nephrectomy in the management of anatomically complex renal masses. Eur Urol. 2017;72(1):64-75.

Publisher's Note Springer Nature remains neutral with regard to jurisdictional claims in published maps and institutional affiliations. 